\title{
Rough Sets and Near Sets in Medical Imaging: A Review
}

\author{
Aboul Ella Hassanien*, Ajith Abraham, Senior Member, IEEE, James F. Peters, Member, IEEE, \\ Gerald Schaefer, Member, IEEE, Christopher Henry, Member, IEEE,
}

\begin{abstract}
This paper presents a review of the current literature on rough set and near set-based approaches to solving various problems in medical imaging such as medical image segmentation, object extraction and image classification. Rough set frameworks hybridized with other computational intelligence technologies that include neural networks, particle swarm optimization, support vector machines and fuzzy sets are also presented. In addition, a brief introduction to near sets and near images with an application to MRI images is given. Near sets offer a generalization of traditional rough set theory and a new approach to classifying perceptual objects by means of features in solving medical imaging problems. Challenges to be addressed and future directions of research are identified and an extensive bibliography is also included.
\end{abstract}

Index Terms-Computational Intelligence, Rough Sets, Near Sets, Medical Imaging, Image Segmentation, Image Classification, Hybrid Rough Image Processing

\section{INTRODUCTION}

Computational intelligence techniques and approaches encompass various paradigms dedicated to approximately solving real-world problems in decision making, pattern classification and learning [1], [2], [3]. Prominent among these paradigms are fuzzy sets, neural networks, genetic algorithms, rough sets, and a generalization of rough sets called near sets. Fuzzy sets provide a natural framework for dealing with uncertainty. It offers a problem-solving tool between the precision of classical mathematics and the inherent imprecision of the real world. For example, imprecision in a segmented image can be represented and analyzed using fuzzy sets. Neural networks provide a robust approach to approximating realvalued, discrete-valued and vector-valued functions. The wellknown back propagation algorithm that uses gradient descent to tune network parameters to best fit the training set with

*A.E. Hassanien is with the Information Technology Department, FCI, Cairo University, 5 Ahamed Zewal Street, Orman, Giza, Egypt, and System Department, CBA, Kuwait University, Kuwait, a.hassanien@fci-cu.edu.eg, abo@cba.edu.kw

A. Abraham is with the Center for Quantifiable Quality of Service in Communication Systems, Norwegian University of Science and Technology O.S. Bragstads plass 2E, N-7491 Trondheim, Norway

J.F. Peters is with the Computational Intelligence Laboratory, Department of Electrical \& Computer Engineering, University of Manitoba, Winnipeg, Manitoba R3T 5V6 Canada

G. Schaefer is with the School of Engineering and Applied Science, Aston University Birmingham, U.K.

C. Henry is a Ph.D. student with the Computational Intelligence Laboratory, Department of Electrical \& Computer Engineering, University of Manitoba, Winnipeg, Manitoba R3T 5V6 Canada input-output pair, has been successfully applied to a variety of problems. Genetic algorithms [3] are stochastic search techniques based on the principles of evolution. Extensive research has been performed exploiting the robust properties of genetic algorithms and demonstrating their capabilities across a broad range of problems. These evolutionary methods have gained recognition as general problem solving techniques in many applications, including function optimization, image processing, classification and machine learning, training of neural networks, and system control. Other approaches like case based reasoning and decision trees [4], [5] are also widely used to solve data analysis problems.

Recently, various published algorithms have been applied to build a computer-aided analysis system in the medical field [6], [7]. The most commonly used algorithms are neural networks, Bayesian classifiers, genetic algorithms, decision trees, and fuzzy theory [8], [9], [10], [11], [12]. Unfortunately, the techniques developed have not been sufficient to introduce an effective computer-aided analysis in clinical use. A survey of the area can be found in [6].

Rough set theory introduced by Pawlak during the early 1980s [13] spans a quarter century (see, e.g., [14], [15], [16], [17]). The rough set approach to approximation of sets leads to useful forms of granular computing that is part of computational intelligence [3]. The basic idea underlying the rough set approach to information granulation is to discover to what extent a given set of objects (e.g., pixel windows in an image) approximates another set of objects of interest. Objects are compared by considering their descriptions. A recent generalization of rough set theory has led to the introduction of near sets [18], [19], [20] and a consideration of the affinities (nearness) of objects [21]. In a near set approach to object classification, an object description is modeled as a vector function values that represent object features [20]. Included in the near set approach is a provision for an object feature to be represented by one or more functions, e.g., colour represented by functions that measure luminance (intensity), type of colour (hue), and purity of colour (saturation).

Near sets and rough sets are very much like two sides of the same coin. From a rough set point-of-view, the focus is on the approximation of sets with non-empty boundaries. By contrast, in a near set approach, the focus is on the discovery of sets having matching descriptions that does not require a consideration approximation boundaries. In the context of medical image analysis, an image is viewed as a set of points. That assumption ushers in either a rough set or near set approach to medical image analysis. There are a 
number of practical outcomes of the near set approach, e.g., feature selection [22], [23], [24], objective evaluation of image segmentations [25], object recognition in images [26], [19], granular computing [27] and in various forms of machine learning [27], [28].

The objective of this article is to present to the rough set and medical imaging research communities the state-of-the-art in rough set-based applications to image processing and pattern recognition in general, and to medical imaging in particular, and motivate research in new trend-setting directions. We review and discuss some representative methods to provide inspiring examples to illustrate how rough sets can be applied to resolve medical imaging problems and how medical images can be analyzed, processed, and characterised by rough sets. These examples include (i) rough representation of a region of interest; (ii) rough image entropy; (iii) rough C-means clustering; and (iv) rough neural intelligent approach for image classification.

This article has the following organization. To provide useful insights for rough set applications in medical imaging, we structure the rest of this paper in six further sections, where Section II provides an explanation of the basic framework of rough set theory, along with some of the key definitions. Section III provides an introduction to rough image processing including rough images, rough representation of a region of interest, rough image entropy, and rough-based medical image applications including object extraction and medical image segmentation and clustering. Some useful measures are presented in Section IV. Section V provides a brief review of rough sets combined with other computational intelligence approaches such as rough neural networks, rough fuzzy and rough genetic algorithms as well as Bayesian methods, particle swarm optimization and support vector machines coupled with rough sets. An introduction to near sets, near images and the near set approach to image segmentation is given in Section VI. Finally. challenges and future trends are discussed in Section VIII.

\section{ROUGH SETS: FOUNDATIONS}

Due to space limitation we provide only a brief explanation of the basic framework of rough set theory, along with some of the key definitions. A more comprehensive review can be found in sources such as [14].

Rough sets theory provides a novel approach to knowledge description and to approximation of sets. Rough theory was introduced by Pawlak during the early 1980s [13] and elaborated in [13], [14]. It is based on an approximation space-based approach to classifying sets of objects. In rough sets theory, feature values of sample objects are collected in what are known as information tables. Rows of a such a table correspond to objects and columns correspond to object features. Let $\mathcal{O}, \mathcal{F}$ denote a set of sample objects and a set of functions representing object features, respectively. Assume that $B \subseteq \mathcal{F}, x \in \mathcal{O}$. Further, let $x_{\sim_{B}}$ denote

$$
x_{/ \sim_{B}}=\{y \in \mathcal{O} \mid \forall \phi \in B, \phi(x)=\phi(y)\},
$$

i.e., $x \sim_{B} y$ (description of $x$ matches the description of $y$ ). Rough sets theory defines three regions based on the equivalent classes induced by the feature values: lower approximation $\underline{B} X$, upper approximation $\bar{B} X$ and boundary $B N D_{B}(X)$. A lower approximation of a set $X$ contains all equivalence classes $x /_{\sim_{B}}$ that are proper subsets of $X$, and upper approximation $\bar{B} X$ contains all equivalence classes $x_{/ \sim_{B}}$ that have objects in common with $X$, while the boundary $B N D_{B}(X)$ is the set $\bar{B} X \backslash \underline{B} X$, i.e., the set of all objects in $\bar{B} X$ that are not contained in $\underline{B} X$. Any set $X$ with a non-empty boundary is roughly known relative, i.e., $X$ is an example of a rough set.

The indiscernibility relation $\sim_{B}$ (or by $\operatorname{Ind} d_{B}$ ) is a mainstay of rough set theory. Informally, $\sim_{B}$ is a set of all classes of objects that have matching descriptions. Based on the selection of $B$ (i.e., set of functions representing object features), $\sim_{B}$ is an equivalence relation partitions a set of objects $\mathcal{O}$ into classes (also called elementary sets [13]). The set of all classes in a partition is denoted by $\mathcal{O}_{\sim_{\sim_{B}}}$ (also by $\mathcal{O} / \operatorname{Ind}_{B}$ ). The set $\mathcal{O} / \operatorname{Ind}_{B}$ is called the quotient set. Affinities between objects of interest in the set $X \subseteq \mathcal{O}$ and classes in a partition can be discovered by identifying those classes that have objects in common with $X$. Approximation of the set $X$ begins by determining which elementary sets $x_{/ \sim_{B}} \in \mathcal{O}_{/ \sim_{B}}$ are subsets of $X$.

\section{Rough Image Processing}

Various rough image processing (RIP) methodologies have been applied to handle the different challenges posed by medical imaging. We can define the RIP as the collection of all approaches and techniques that understand, represent and process the images, their segments and features as rough sets (see, e.g., [29], [10], [30], [31]). In this section, we first describe the ability of rough sets to handle and represent images and color images, followed by the various rough based approaches developed for handling the different functional aspects to solve medical imaging problems.

\section{A. The ability of rough sets to handle images}

Rough sets provide reasonable structures for the overlap boundary given domain knowledge. The case study for images of the heart on cardiovascular magnetic resonance (MR) images also extends to handling multiple types of knowledge including: myocardial motion, location and signal intensity. A study concerned with distinguishing different picture types of the central nervous system is introduced in [32]. Research involving color images appears in [33]. Histones (i.e., encrustations of a histogram) are used as the primary measure and as a visualization of multi-dimensional color information. The basic idea of a histon is to build a histogram on top of the histograms of the primary color components red, green, and blue. The authors show that the base histogram correlates with the lower approximation, whereas the encrustation correlates with the upper approximation. The problem of a machine vision application where an object is imaged by a camera system is considered in [34]. The object space can be modeled as a finite subset of the Euclidean space when the objects image is captured via an imaging system. Rough sets can bound such sets and provide a mechanism for modeling the 
spatial uncertainty in the image of the object. This work introduced a rough sets approach for building pattern matching systems that can be applicable with a wide range of images in medical sciences.

\section{B. Rough images}

In gray scale images boundaries between object regions are often ill defined because of grayness or spatial ambiguities [35], [36]. This uncertainty can be effectively handled by describing the different objects as rough sets with upper (or outer) and lower (or inner) approximations. Here the concepts of upper and lower approximation can be viewed, respectively, as outer and inner approximations of an image region in terms of granules [36] defined the rough as an image as follows:

Definition 1: (Rough image) Let the universe $U$ be an image consisting of a collection of pixels. Then if we partition $U$ into a collection of non-overlapping windows of size $m \times n$, each window can be considered as a granule $G$. Given this granulation, object regions in the image can be approximated by rough sets.

A rough image is a collection of pixels and the equivalence relation induced partition of an image into sets of pixels lying within each non-overlapping window over the image. With this definition, the roughness of various transforms (or partitions) of an image can be computed using image granules for windows of different sizes.

\section{Rough representation of a region of interest}

A Region of Interest, often abbreviated ROI, is a selected subset of samples within an image identified for a particular purpose. For example: the boundaries of an object in 2D images and the contours or surfaces outlining an object in a volume dataset. The concept of an $R O I$ is commonly used in medical imaging. For example, the boundaries of a tumor may be defined on an image or in a volume, for the purpose of measuring its size. The endocardial border may be defined on an image, perhaps during different phases of the cardiac cycle, say end-systole and end-diastole, for the purpose of assessing cardiac function.

Hirano and Tsumoto [37] introduced the rough direct representation of ROIs in medical images. The main advantage of this method is its ability to represent inconsistency between the knowledge-driven shape and image-driven shape of a $R O I$ using rough approximations. The method consists of three steps. First, they derive discretized feature values that describe the characteristics of a ROI. Secondly, using all features, they build up the basic regions (namely categories) in the image so that each region contains voxels that are indiscernible on all features. Finally, according to the given knowledge about the ROI, they construct an ideal shape of the ROI and approximate it by the basic categories. Then the image is split into three regions: a set of voxels that are:

(1) certainly included in the ROI (Positive region),

(2) certainly excluded from the $R O I$ (Negative region),

(3) possibly included in the $R O I$ (Boundary region).

The $R O I$ is consequently represented by the positive region associated with some boundary regions. In the experiments we

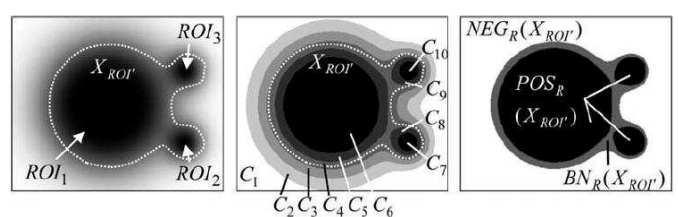

Fig. 1. Rough ROI representation. Left: an original image. Middle: elementary categories C1C9. Right: roughly segmented ROI [37]

show the result of implementing a rough image segmentation system.

Hirano and Tsumoto [29], [37] described the procedures for rough representation of ROIs under single and multiple types of classification knowledge. Usually, the constant variables defined in the prior knowledge, for example some threshold values, do not meet the exact boundary of images due to inter-image variances of the intensity. The approach tries to roughly represent the shape of the $R O I$ by approximating the given shapes of the $R O I$ by the primitive regions derived from feature of the image itself. It is reported that the simplest case where we have only information about intensity range of the ROI. In this case intensity thresholding is a conventional approach to obtain the voxels that fall into the given range. Let us denote the lower and upper thresholds by $T h L$ and $T h H$, respectively. Then the $R O I$ can be represented by:

$$
R O I=\left\{x(p) \mid T h_{L} \leq I(x) P \leq T h_{P}\right\},
$$

where $x(p)$ denotes a voxel at location $p$ and $I(x(p))$ denotes intensity of voxel $x(p)$.

Figure 1 illustrates the concept of rough $R O I$ representation. The left image is an original grayscale image. Assume that the ROIs are three black circular regions: $R O I_{1}, R O I_{2}$, and $\mathrm{ROI}_{3}$. Also assume that we are given a prior knowledge about the $R O I s$, that is, the lower threshold value $T h_{L}$ of the ROIs, derived from some knowledge base. With this knowledge we can segment an ideal $R O I X_{R \hat{O} I}$ as follows:

$$
X_{R \hat{O} I}=\left\{x(p) \mid T h_{L} \leq I(p)\right\} .
$$

However, $X_{R O \hat{I}}$ does not correctly match the expected ROIs. This is because $T h_{L}$ was too small to separate the ROIs. $T h_{L}$ is a global threshold determined on the other sets, therefore, it should not be directly applied to this image.

\section{Rough Image Entropy}

Entropy-based information theoretic approaches have received considerable interest in image analysis approaches such as image registration [38]. Previous work on entropic thresholding is based on Shannon's entropy. The idea is to calculate Shannon's entropy based on a co-occurrence matrix and use it as a criterion for selecting an appropriate threshold value. The approach using relative entropy for image thresholding has been shown very competitive compared to Pal's methods, where the relative entropy is chosen to be a thresholding criterion of measuring mismatch between an image and a thresholded image. Currently there are various published approaches using relative entropy and applying 


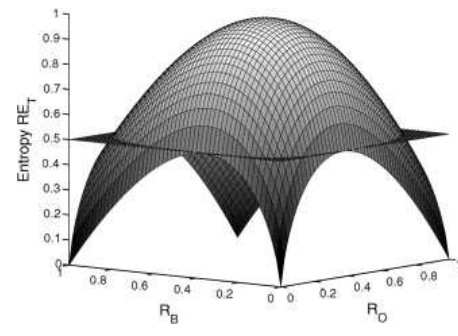

Fig. 2. rough entropy for various values [36]

to medical images, multispectral imagery, temporal image sequences, multistage thresholding and segmentation.

Pal et al. [36] presented a new definition of image entropy in a rough set theoretic framework, and its application to the problem of object extraction from images by minimizing both object and background roughness. Granules carry local information and reflect the inherent spatial relation of the image by treating pixels of a window as indiscernible or homogeneous. Maximization of homogeneity in both object and background regions during their partitioning is achieved through maximization of rough entropy; thereby providing optimum results for object background classification.

Definition 2: (Rough Image Entropy)[36] Rough image entropy $\left(R_{I} E\right)$ is defined by:

$$
R_{I} E=-\frac{e}{2}\left[R_{O_{T}} \log _{e}\left(R_{O_{T}}\right)+R_{B_{T}} \log _{e}\left(R_{B_{T}}\right)\right] .
$$

Pal [36] noted that the value of $R_{I} E$ lies between 0 and 1 and it has has a maximum value of unity when $R_{O_{T}}=R_{B_{T}}=\frac{1}{e}$, and minimum value of zero when $R_{O_{T}}, R_{B_{T}} \in\{0,1\}$.

Figure 2 shows a sample plot of rough entropy for various values of roughness of the object and background [36].

Pal et al. [36] reported that a maximization of homogeneity in both object and background regions during their partitioning is achieved through maximization of rough entropy; thereby providing optimum results for object-background classification. Also, maximization of the rough entropy measure minimizes the uncertainty arising from vagueness of the boundary region of the object. Therefore, for a given granule size, the threshold for object-background classification can be obtained through its maximization with respect to different image partitions. The rough entropy concepts is applicable for many medical imaging problems such as feature extraction and medical image segmentation problems.

\section{E. Rough Sets for Object Extraction}

Identification of anatomical features is a necessary step for medical image analysis. Automatic methods for feature identification using conventional pattern recognition techniques typically classify an object as a member of a predefined class of objects, but do not attempt to recover the exact or approximate shape of that object. For this reason, such techniques are usually not sufficient to identify the borders of organs when individual geometry varies in local detail, even though the general geometrical shape is similar.

Pal et al. [36] demonstrated a new application of rough sets for object extraction from gray scale image. In gray scale images boundaries between object regions are often illdefined. This uncertainty can be handled by describing the different objects as rough sets with upper (outer) and lower (inner) approximations. The set approximation capability of rough sets is exploited in the present investigation to formulate an entropy measure, called rough entropy, quantifying the uncertainty in an object-background image. Let $T$ denote a set of thresholds. An image object and background are viewed as two sets with their rough representation by computing the inner approximation of the object $\left(\underline{Q}_{T}\right)$, outer approximation of the object $\left(\bar{Q}_{T}\right)$, inner approximation of the background $\left(\underline{B}_{T}\right)$ and outer approximation of the background $\left(\bar{B}_{T}\right)$ as follows:

$$
\begin{gathered}
\underline{Q}_{T}=\bigcup G_{i} \mid p_{j}>T, \forall j=1, \ldots, m n, \\
\bar{Q}_{T}=\bigcup G_{i}, \exists j, p_{j}>T, j=1, \ldots, m n, \\
\underline{B}_{T}=\bigcup G_{i} \mid p_{j}>T, \forall j=1, \ldots, m n, \\
\bar{B}_{T}=\bigcup G_{i}, \exists j, p_{j} \leq T, j=1, \ldots, m n,
\end{gathered}
$$

where $p_{j}$ is a pixel in $G_{i}$. The rough set representation of the image for a given $I_{m \times n}$ depends on the value of $T$.

Pal et al. [36] define the roughness (R) of the object $O_{T}$ and the background $B_{T}$ as follows:

$$
\begin{aligned}
& R_{O_{T}}=1-\frac{\left|\underline{Q}_{T}\right|}{\left|\bar{Q}_{T}\right|}, \\
& R_{B_{T}}=1-\frac{\left|\underline{B}_{T}\right|}{\left|\bar{B}_{T}\right|},
\end{aligned}
$$

where $|S|$ is the cardinality of the set. The presented method may be applicable for many application in image processing, in particulars in medical imaging problems such as automatically identify the myocardial contours of the heart,segmentation of knee tissues in CT image and segmentation of brain tissues in MR images.

\section{F. Rough Sets in Medical Image Segmentation}

The basic idea behind segmentation-based rough sets is that while some cases may be clearly labelled as being in a set $X$ called positive region in rough sets theory), and some cases may be clearly labelled as not being in set $X$ called negative region in rough sets theory, limited information prevents us from labelling all possible cases clearly. The remaining cases cannot be distinguished and lie in what is known as the boundary region. A little bit effort has been done in uses the rough sets in image segmentation and in particulars in medical segmentation problems.

Among many difficulties in segmenting MRI data, the partial volume effect (PVE) arises in volumetric images when more than one tissue type occurs in a voxel. In such cases, the voxel intensity depends not only on the imaging sequence and tissue properties, but also on the proportions of each tissue type present in the voxel. Sebastian et al. [11] discussed the partial volume effect problem in the segmentation of magnetic resonance imaging data that entails assigning tissue class labels to voxels. They employ rough sets to identify 
automatically the partial volume effect, which occurs most often with low resolution imaging.

An interesting strategy for color image segmentation using rough set theory has been presented by Mohabey et al. [33]. A new concept of encrustation of the histogram, called histon, has been proposed for the visualization of multi-dimensional color information in on integrated fashion and its applicability in boundary region analysis has been shown. The histon correlates with the upper approximation of a set such that all elements belonging to this set are clarified as possibly belonging to the same segment or segments showing similar color value. The proposed encrustation provides a direct means of segregating pool of inhomogeneous regions into its components. Experimental results for various images have been presented in their work. They also introduced a hybrid rough set theoretic approximations and fuzzy c-means algorithm for color image segmentation. They segmented natural images with regions having gradual variations in color value. The technique extracts color information regarding the number of segments and the segments center values from the image itself through rough set theoretic approximations and presented it as input to fuzzy c-means block for the soft evaluation of the segments. The performance of the algorithm has been evaluated on various natural and simulated images.

Many clustering algorithms [39] have been developed and applied in medical imaging problems, while most of them cannot process objects in hybrid numerical/nominal feature space or with missing values. In many of them, the number of clusters has to be manually specified while the clustering results are sensitive to the input order of the objects to be clustered. This clearly limits their applicability and reduces the quality of clustering. To solve this problem, an improved clustering algorithm based on rough set (RS) and entropy theory was presented by Chena and Wang [40] which aims to avoid the need to pre-specify the number of clusters while also allowing clustering in both numerical and nominal feature space with the similarity introduced to replace the distance index. At the same time, the RS theory endows the algorithm with the function to deal with vagueness and uncertainty in data analysis. Shannon's entropy was used to refine the clustering results by assigning relative weights to the set of features according to the mutual entropy values. A novel measure of clustering quality was also presented to evaluate the clusters. The experimental results confirm that performances of efficiency and clustering quality of this algorithm are improved.

Widz et al. [11] introduced an automated multi-spectral MRI segmentation technique based on approximate reducts derived from the theory of rough sets. They utilized the $T 1, T 2$ and $P D$ MRI images from the simulated Brain Database as a gold standard to train and testing their segmentation algorithm. The results suggest that approximate reducts, used alone or in combination with other classification methods, may provide a novel and efficient approach to the segmentation of volumetric MRI data sets. Segmentation accuracy reaches $96 \%$ for the highest resolution images and $89 \%$ for the noisiest image volume. They tested the resultant classifier on real clinical data, which yielded an accuracy of approximately $84 \%$.

\section{G. Adaptation of C-Means to Rough Set Theory}

$C$-means clustering is an iterative technique that is used to partition an image into $c$ clusters. Fuzzy c-means (FCM) is one of the most commonly used fuzzy clustering techniques for different degree estimation problems, especially in medical image processing [41]. Lingras [42] described modifications of clustering based on Genetic Algorithms, k-means algorithm, and Kohonen Self-Organizing Maps (SOM). These modifications make it possible to represent clusters as rough sets.

$\mathrm{K}$-means clustering is one of the most popular statistical clustering techniques used in segmentation of medical images [43], [44], [45]. The name K-means originates from the means of the $k$ clusters that are created from $n$ objects. Let us assume that the objects are represented by $m$-dimensional vectors. The objective is to assign these $n$ objects to $k$ clusters. Each of the clusters is also represented by an m-dimensional vector, which is the centroid or mean vector for that cluster. The process begins by randomly choosing $k$ objects as the centroids of the $k$ clusters. The objects are assigned to one of the $k$ clusters based on the minimum value of the distance $d(v, x)$ between the object vector $v=\left(v_{1}, \ldots, v_{j}, \ldots, v_{m}\right)$ and the cluster vector $x=\left(x_{1}, \ldots, x_{j}, \ldots, x_{m}\right)$. After the assignment of all the objects to various clusters, the new centroid vectors of the clusters are calculated as:

$$
x_{j}=\frac{\sum_{v \in x} v_{j}}{S O C}, 1 \leq j \leq m,
$$

where $S O C$ is the size of cluster $x$. Lingras [42] observes that incorporating rough sets in $\mathrm{K}$-means clustering requires the addition of the concept of lower and upper bounds. Calculation of the centroids of clusters from conventional k-means needs to be modified to include the effects of lower as well as upper bounds. The modified centroid calculations for rough sets are then given by:

$$
c e n_{j}=w_{l o w} \times \frac{\sum_{v \in R(x)}}{\mid \underline{R(x) \mid}}+w_{u p} \times \frac{\sum_{v \in\left(B N_{R}(x)\right)}}{\underline{B N_{R}(x) \mid}},
$$

where $1 \leq j \leq m$. The parameters $w_{l o w}$ and $w_{u p}$ correspond to the relative importance of lower and upper bounds, and $w_{\text {low }}+w_{\text {up }}=1$. If the upper bound of each cluster were equal to its lower bound, the clusters would be conventional clusters. Therefore, the boundary region $B N_{R}(x)$ will be empty, and the second term in the equation will be ignored. Thus, the above equation will reduce to conventional centroid calculations. The next step in the modification of the $K$ means algorithms for rough sets is to design criteria to determine whether an object belongs to the upper or lower bound of a cluster.

\section{H. Rough Sets in Feature Reduction and Image Classification}

Many researchers have endeavored to develop efficient and effective algorithms to compute useful feature extraction and reduction of information systems and besides mutual information and discernibility matrix based feature reduction methods. These techniques have been successfully applied to medical domain [46], [47].

Wojcik [30] approached the nature of a feature recognition process through the description of image features in terms of 
rough sets. Since the basic condition for representing images must be satisfied by any recognition result, elementary features are defined as equivalence classes of possible occurrences of specific fragments existing in images. The names of the equivalence classes (defined through specific numbers of objects and numbers of background parts covered by a window) constitute the best lower approximation of window contents (i.e., names of recognized features). The best upper approximation is formed by the best lower approximation, its features, and parameters, all referenced to the object fragments situated in the window. The rough approximation of shapes is resistant to accidental changes in the width of contours and lines and to small discontinuities and, in general, to possible positions or changes in shape of the same feature. The rough sets are utilized also on the level of image processing for noiseless image quantization. This initiative study is very interest in many area of medical image processing including filtering, segmentation and classification.

Swiniarski and Skowron [48] presented applications of rough set methods for feature selection in pattern recognition. They emphasize the role of the basic constructs of rough set approach in feature selection, namely reducts and their approximations, including dynamic reducts. Their algorithm for feature selection is based on an application of a rough set method to the result of principal components analysis (PCA) used for feature projection and reduction. They present various experiments including face and mammogram recognition.

Hu et al. [49] proposed an information measure to computing discernibility power of a crisp equivalence relation or a fuzzy one, which is the key concept in classical rough set model and fuzzy-rough set model. Based on the information measure, a general definition of significance of nominal, numeric and fuzzy features is presented.

Lymphoma is a broad term encompassing a variety of cancers of the lymphatic system and is differentiated by the type of cell that multiplies and how the cancer presents itself. It is very important to get an exact diagnosis regarding lymphoma and to determine the treatments that will be most effective for the patient's condition. Milan et al. [50] focused on the identification of lymphoma by finding follicles in microscopy images. Their study comprises two stages: in the first stage they did image pre-processing and feature extraction, while in the second stage they used different rough set approaches for pixel classification. These results were compared to decision tree results. The results they got are very promising and show that symbolic approaches can be successful in medical image analysis applications.

Microcalcification on x-ray mammogram is a significant mark for early detection of breast cancer. Texture analysis methods can be applied to detect clustered microcalcification in digitized mammograms. In order to improve the predictive accuracy of the classifier, the original number of feature set is reduced into smaller set using feature reduction techniques. Thangavel et al. [51] introduced rough set based reduction algorithms such as Decision Relative Discernibility based reduction, Heuristic approach, Hu's algorithm, Quick Reduct $(\mathrm{QR})$, and Variable Precision Rough Set (VPRS) to reduce the extracted features. The performance of all the introduced algorithms is compared. The Gray Level Co-occurrence Matrix (GLCM) is generated for each mammogram to extract the Haralick features as feature set.

Cyran et al. [52] showed how rough sets can be applied to improve the classification ability of a hybrid pattern recognition system. The system presented consists of a feature extractor based on a computer-generated hologram (CGH). Features extracted are shift, rotation, and scale invariant and although they can be optimized. This article presented an original method of optimizing the feature extraction abilities of a $\mathrm{CGH}$.

Jiang et al. [53] developed a joining associative classifier (JAC) algorithm using the rough set theory to mining digital mammography images. The experimental results showed that the joining associative classifier performance at $77.48 \%$ of classifying accuracy which is higher than $69.11 \%$ using associative classifier only. At the same time, the number of rules decreased distinctively.

\section{Quantitative Evaluation}

This section presents some quantitative measures [54] that are capable of quantifying the relative utility of enhancement techniques in digital imaging, generated rules and quality of classification measures [55], [56]. This relates to preference criteria and goodness-of-fit chosen for the rules and classifiers.

A key objective of contrast enhancement is to maximize the difference between the background mean and target mean grey scale level and ensure that the homogeneity of the mass is increased aiding the visualization of its boundaries and location. Using the ratio of the standard deviation of the grey scales within the target before and after enhancement, we can quantify this improvement using the target-to-background contrast enactment based on the standard deviation. This measure is initially computed by determining the difference between ratios of the mean grey scales in the target and background images in the original and enhances images in (12).

$$
C M_{S D}=\left\{\frac{\left(m_{t}^{e} / m_{b}^{e}\right)-\left(m_{t}^{o} / m_{b}^{o}\right)}{\sigma_{t}^{e} / \sigma_{t}^{o}}\right\}
$$

where $m_{t}^{e}, m_{b}^{e}, m_{t}^{o}, m_{b}^{o}$ are the mean of the grey scales comprising the target and background respectively of the original image before and after enhancement and where $\sigma_{t}^{e}, \sigma_{t}^{o}$ the standard deviations of the grey scales are before and after enhancement.

Within the mammogram image, the target has a greater density within the mammogram thus having higher mean grey scale intensity compared to the surrounding background. A good enhancement algorithm should aim to enhance the contrast between target and background by increasing the mean grey scale of the target area and then reducing the mean grey of the background area, thereby increasing the value of $C M_{S D}$.

The background contrast ratio can also be calculated using the entropy $E$ of target and background areas within an image. This measure is computed in a similar manner to $C M_{S D}$ by determining the difference between ratios of the mean grey scales in the target and background areas in both original and 
enhanced images in (13).

$$
C M_{\text {Entropy }}=\left\{\frac{\left(m_{t}^{e} / m_{b}^{e}\right)-\left(m_{t}^{o} / m_{b}^{o}\right)}{E_{t}^{e} / E_{t}^{o}}\right\},
$$

where $E_{t}^{e}$ and $E_{t}^{o}$ are the entropy of the target in the original and enhancement image, respectively. An effective enhancement algorithm will lead to a large value of $C M_{\text {Entropy }}$.

Index of fuzziness and fuzzy entropy are measures for global greyness ambiguity (fuzziness) of an image. They can be regarded as a degree of difficulty in deciding whether a pixel would be treated as black (dark) or white (bright). The index of fuzziness that gives the amount of fuzziness present in an image determines the amount of vagueness by measuring the distance between its fuzzy property plane and the nearest ordinary plane. Accordingly, entropy, $H$ which makes use of Shannon's function, is regarded as a measure of quality of information in an image in the fuzzy domain. It gives the value of indefiniteness of an image. These quantities [57], [58], [59], [60] are defined in (14) and (15).

$$
\begin{gathered}
\gamma=\frac{2}{M N} \sum_{M} \sum_{N} \min \left(\mu_{m n}, 1-\mu_{m n}\right), \\
H=\frac{1}{M N} \ln \sum_{M} \sum_{N} \ln \left(\mu_{m n}\right)-\left(1-\mu_{m n}\right) \cdot \ln \left(1-\mu_{m n}\right) .
\end{gathered}
$$

It should be noted that the decrease in the index of fuzziness and fuzzy entropy does not ensure proper enhancement of the images. We can only say that a good enhancement algorithm should reduce the greyness ambiguity. However, a low amount of ambiguity does not automatically lead to the desired enhancement effect.

\section{Hybrid COMPUTATIONALly INTELLIGENT APPROACHES}

Intelligent systems comprise various paradigms dedicated to approximately solving real-world problems, e.g., in decision making, classification or learning; among these paradigms are fuzzy sets, neural networks, decision tree, and rough sets, algorithms. Combination of kinds of computational intelligence techniques in application area of pattern recognition and in particulars in medical imaging problems has become one of the most important ways of research of intelligent information processing [61].

\section{A. Neural Networks with Rough sets in medical imaging}

Neural network shows us its strong ability to solve complex problems for medical image processing. But neural network are unable to identify redundant information from huge amount of data, which will easily lead to some problems such as too complex network structure, long training time, and low convergence speed. These problems have been addressed and discussed to solve for medical imaging problems such as detecting tumors in mammography images. This problem is a difficult task because of the complexity of the images. This brings the necessity of creating automatic tools to find whether a mammogram contains a tumor or not. For example,
Hassanien and Ślęzak [54] introduced a rough neural approach for rule generation and image classification. Hybridization of intelligent computing techniques has been applied to see their ability and accuracy to classify breast cancer images into malignant and benign cases. Algorithms based on fuzzy image processing are first applied to enhance the contrast of the whole original image; to extract the region of interest and to enhance the edges surrounding that region. Then, they extract features characterizing the underlying texture of the regions of interest by using the grey-level co-occurrence matrix. Then, the rough set approach to feature reduction and rule generation is presented. Finally, rough neural network is designed for discrimination of different regions of interest to test whether they represent malignant cancer or benign cancer. Rough neural network is built from rough neurons, each of which can be viewed as a pair of sub-neurons, corresponding to the lower and upper bounds. To evaluate performance of the presented rough neural approach, they run tests over different mammogram images. In their experiments, results show that the overall classification accuracy offered by rough neural approach is high compared with other intelligent techniques.

The introduced rough neural networks [62], [63], [64] used in their study, consist of one input layer, one output layer and one hidden layer. The input layer neurons accept input from the external environment. The outputs from input layer neurons are fed to the hidden layer neurons. The hidden layer neurons feed their output to the output layer neurons which send their output to the external environment.

The number of hidden neurons is determined by the following inequality [65].

$$
N_{h n} \leq \frac{N_{t s} * T_{e} * N_{f}}{N_{f}+N_{o}}
$$

$N_{h n}$ is the number of hidden neurons, $N_{t s}$ is the number of training samples, $T_{e}$ is the tolerance error, $N_{f}$ is the number of features, and $N_{o}$ is the number of the output.

The output of a rough neuron is a pair of upper and lower bounds, while the output of a conventional neuron is a single value. Rough neuron was introduced in 1996 by Lingras [62]. It was defined relative to upper bound $\left(U_{n}\right)$, lower bound $\left(L_{n}\right)$, and inputs were assessed relative to boundary values. Rough neuron has three types of connections:

Step 1. Input-Output connection to $U_{n}$

Step 2. Input-Output connection to $L_{n}$

Step 3. Connection between $U_{n}$ and $L_{n}$

Definition 3: (Rough neuron) A rough neuron $R_{n}$ is a pair of usual rough neurons $R_{n}=\left(U_{n}, L_{n}\right)$, where $U_{n}$ and $L_{n}$ are the upper rough neuron and the lower rough neuron, respectively.

Let $\left(\operatorname{Ir}_{L_{n}}, O r_{L_{n}}\right)$ be the input/output of a lower rough neuron and $\left(I r_{U_{n}}, O r_{U_{n}}\right)$ be the input/output of an upper rough neuron. Calculation of the input/output of the lower/upper rough neurons is given as follows:

$$
I r_{L_{n}}=\sum_{j=1}^{n} w_{L_{n j}} O n_{j}
$$




$$
\begin{gathered}
\operatorname{Ir}_{U_{n}}=\sum_{j=1}^{n} w_{U_{n j}} O n_{j}, \\
O r_{L_{n}}=\min \left(f\left(\operatorname{Ir}_{L_{n}}\right), f\left(\operatorname{Ir}_{U_{n}}\right)\right), \\
O r_{U_{n}}=\max \left(f\left(\operatorname{Ir}_{L_{n}}\right), f\left(\operatorname{Ir}_{U_{n}}\right)\right) .
\end{gathered}
$$

The output of the rough neuron $\left(O_{r n}\right)$ will be computed as follows:

$$
O_{r n}=\frac{O r_{U_{n}}-O r_{L_{n}}}{\text { average }\left(O r_{U_{n}}, O r_{L_{n}}\right)} .
$$

In their experiments, the segmentation performance is measured by the value of accuracy as defined below and the average of segmentation accuracy achieved by the reported algorithm is $97 \%$, which means that it is robust enough.

Another success example introduced by Jiang et al. [66] which integrate neural network with reduction of rough set theory which they called the rough neural network (RNN) to classify digital mammography. Their experimental results showed that the RNN performs better than purely using neural network in terms of time, and it can get $92.37 \%$ classifying accuracy which is higher than $81.25 \%$ using neural network only.

Swiniarski and Hargis [48] described an application of rough sets method to feature selection and reduction as a front end of neural-network-based texture images recognition. The methods applied include singular-value decomposition (SVD) for feature extraction, principal components analysis (PCA) for feature projection and reduction, and rough sets methods for feature selection and reduction. For texture classification the feedforward backpropagation neural networks were applied. The numerical experiments showed the ability of rough sets to select reduced set of pattern's features, while providing better generalization of neural-network texture classifiers, see also [46].

\section{B. Rough-fuzzy hybridization in medical imaging}

Also defined in the literature are rough-fuzzy sets [67], which can be seen to be a particular case of fuzzy-rough sets. A rough-fuzzy set is a generalisation of a rough set derived from the approximation of a fuzzy set in a crisp approximation space. This corresponds to the case where only the decision attribute values are fuzzy; the conditional values are crisp. The lower and upper approximations indicate the extent to which objects belong to a target set. Mao et al. [68] proposed a new fuzzy Hopfield-model net based on rough-set reasoning for the classification of multispectral images. The main purpose is to embed a rough-set learning scheme into the fuzzy Hopfield network to construct a classification system called a rough-fuzzy Hopfield net (RFHN). The classification system is a paradigm for the implementation of fuzzy logic and rough systems in neural network architecture. Instead of all the information in the image being fed into the neural network, the upper- and lower-bound grey levels, captured from a training vector in a multispectal image, are fed into a rough-fuzzy neuron in the RFHN. Therefore, only $2 / \mathrm{N}$ pixels are selected as the training samples if an $\mathrm{N}$-dimensional multispectral image was used. In the simulation results, the proposed network not only reduces the consuming time but also reserves the classification performance.

Wang et al. [69] proposed a new nearest neighbor clustering classification algorithm based on fuzzy-rough set theory (FRNNC). First, they make every training sample fuzzyroughness and use edit nearest neighbor algorithm to remove training sample points in class boundary or overlapping regions, and then use Mountain Clustering method to select representative cluster center points, then Fuzzy-Rough Nearest neighbor algorithm (FRNN) is applied to classify the test data. The new algorithm is applied to hand gesture image recognition, the results show that it is more effective and performs better than other nearest neighbor methods. The introduced algorithm is recommended to use in any type of medical image application. such as medical images sequences or finding similar tumor shapes.

Hassanien [70] introduced a hybrid scheme that combines the advantages of fuzzy sets and rough sets in conjunction with statistical feature extraction techniques. An application of breast cancer imaging has been chosen and hybridization scheme have been applied to see their ability and accuracy to classify the breast cancer images into two outcomes: cancer or non-cancer. The introduced scheme starts with fuzzy image processing as pre-processing techniques to enhance the contrast of the whole image; to extracts the region of interest and then to enhance the edges surrounding the region of interest. A subsequently extract features from the segmented regions of the interested regions using the grey-level co-occurrence matrix is presented. Rough sets approach for generation of all reducts that contains minimal number of features and rules is introduced. Finally, these rules can then be passed to a classifier for discrimination for different regions of interest to test whether they are cancer or non-cancer. To measure the similarity, a new rough set distance function is presented. The experimental results showed that the hybrid scheme applied in this study perform well reaching over $98 \%$ in overall accuracy with minimal number of generated rules.

Image clustering analysis is one of the core techniques for image indexing, classification, identification and segmentation for medical image processing. Mitra et al. [71] introduced a hybrid clustering architecture, in which several subsets of patterns can be processed together with an objective of finding a common structure. A detailed clustering algorithm is developed by integrating the advantages of both fuzzy sets and rough sets, and a measure of quantitative analysis of the experimental results is provided for synthetic and real-world data. The structure revealed at the global level is determined by exchanging prototypes of the subsets of data and by moving prototypes of the corresponding clusters toward each other. Thereby, the required communication links are established at the level of cluster prototypes and partition matrices, without hampering the security concerns.

Petrosino et al. [72] presented a multi-scale method based on the hybrid notion of rough fuzzy sets, coming from the combination of two models of uncertainty like vagueness by handling rough sets and coarseness by handling fuzzy 
sets. Marrying both notions lead to consider, as instance, approximation of sets by means of similarity relations or fuzzy partitions. The most important features are extracted from the scale spaces by unsupervised cluster analysis, to successfully tackle image processing tasks. Here, we report some results achieved by applying the method to multi-class image segmentation and edge detection, but it can be shown to be successfully applied to texture discrimination problem too. Mitra et al. [71] and Petrosino et al. [72] approaches can be applied in many medical imaging clustering problems such as image segmentation in abdomen medical images and cluster filter bank response vectors to obtain a compact representation of the image structures found within an image quality verification of color retina images in diabetic retinopathy screening, for example.

Sakar [73] generalizes the concept of rough membership functions in pattern classification tasks to rough-fuzzy membership functions and rough-fuzzy ownership functions. Unlike the rough membership value of a pattern, which is sensitive only toward the rough uncertainty associated with the pattern, the rough-fuzzy membership (or ownership) value of the pattern signifies the rough uncertainty as well as the fuzzy uncertainty associated with the pattern. Various set theoretic properties of the rough-fuzzy functions are exploited to characterize the concept of rough-fuzzy sets. These properties are also used to measure the rough-fuzzy uncertainty associated with the given output class. Finally, a few possible applications of the rough-fuzzy functions are included and analyzed.

\section{Bayesian and Particle Swarm Optimization with Rough Sets}

Swiniarski [12] described an application of rough sets and Bayesian inference to a breast cancer detection using electropotentials. The statistical principal component analysis and the rough sets methods were applied for feature extraction, reduction and selection. The quadratic discriminant was applied as a classifier for a breast cancer detection.

Das et al. [8] presented a framework to hybridize the rough set theory with a famous swarm intelligence algorithm known as Particle Swarm Optimization (PSO). The hybrid rough-PSO technique has been used for grouping the pixels of an image in its intensity space. Medical images become corrupted with noise very often. Fast and efficient segmentation of such noisy images (which is essential for their further interpretation in many cases) has remained a challenging problem for years. In there work, they treat image segmentation as a clustering problem. Each cluster is modelled with a rough set. PSO is employed to tune the threshold and relative importance of upper and lower approximations of the rough sets. DaviesBouldin clustering validity index is used as the fitness function, which is minimized while arriving at an optimal partitioning.

Another approach that uses rough set with Particle Swarm Optimization has been proposed in [55]. The authors applied the rough sets to predict the degree of malignancy in brain glioma. As feature selection can improve the classification accuracy effectively, rough set feature selection algorithms are employed to select features. The selected feature subsets are used to generate decision rules for the classification task. A rough set attribute reduction algorithm that employs a search method based on particle swarm optimization (PSO) is proposed and compared with other rough set reduction algorithms. Experimental results show that reducts found by the proposed algorithm are more efficient and can generate decision rules with better classification performance. It is reported that the rough set rule-based method can achieve higher classification accuracy than other intelligent analysis methods such as neural networks, decision trees and a fuzzy rule extraction algorithm based on fuzzy minmax neural networks (FRE-FMMNN). Moreover, the decision rules induced by rough set rule induction algorithm can reveal regular and interpretable patterns of the relations between glioma MRI features and the degree of malignancy, which are helpful for medical experts.

\section{Support vector machines with Rough sets}

Support Vector Machines (SVMs) are a general algorithm based on guaranteed risk bounds of statistical learning theory. They have found numerous applications in image processing and pattern recognition and, in particulars in medical imaging problems such as in classification of brain PET images, detection of microcalcification (MC) clusters in digital mammograms, lung cancer Nodules extraction and classification, etc., and are now established as one of the standard computational intelligence tools. Support vector machines (SVMs) have good classification performances and good capabilities of faulttolerance and generalization. To inherit the merits of both RST and SVMs, a hybrid classifier called rough set support vector machines (RS-SVMs) is proposed by Gexiang et al. [74].

Lingras and Butz [75]described how binary classification with SVMs can be interpreted using rough sets and how rough set theory may help in reducing the storage requirements of the 1-v-1 approach in the operational phase. Their techniques provided better semantic interpretations of the classification process. The theoretical conclusions are supported by experimental findings involving a synthetic dataset. The presented work is useful for soft margin classifiers in solving medical imaging problems especially a multi-class classification system for medical images [76].

In [77], Yun et al. have used a rough-support vector machine integration and developed the Improved Support Vector Machine (ISVM) algorithm to classify digital mammography images, where rough sets are applied to reduce the original feature sets and the support vector machine is used classify the reduced information. The experimental results show that the ISVM classifier can get 96.56 accuracy which is higher about $3.42 \%$ than $92.94 \%$ using SVM, and the error recognition rates are close to $100 \%$ averagely.

\section{Classifying Images: Near Set Approach}

This section gives a brief introduction to a near set approach to classifying images. In this approach, medical images are separated into non-overlapping sets of images that are similar (descriptively near to) each other. The near set approach is well 
suited to investigating medical images. This section introduces recent work on near images by Henry and Peters [26].

Let $\langle O, \mathbb{F}\rangle$ be a perceptual system, i.e., a real valued total deterministic information system where $O$ is a non-empty set of perceptual objects, and $\mathbb{F}$ is a countable set of probe functions. For every $\mathcal{B} \subseteq \mathbb{F}$, the weak nearness relation $\simeq_{\mathcal{B}}$ is defined as follows,

$$
\simeq_{\mathcal{B}}=\left\{(x, y) \in O \times O \mid \exists \phi_{i} \in \mathcal{B}, \phi_{i}(x)=\phi_{i}(y)\right\} .
$$

The relation $\simeq_{\mathcal{B}}$ is considered weak, since this nearness relation between the objects (e.g., pixels in an image) in each pair $(x, y)$ requires at least one (not every) probe function $\phi_{i} \in \mathcal{B}$ such that $\phi_{i}(x)=\phi_{i}(y)$ to establish that $x$ and $y$ are near each other. Furthermore, let $X, Y \subseteq O$. A set $X$ is weakly near to a set $Y$ within the perceptual system $\langle O, \mathbb{F}\rangle\left(X \bowtie_{\mathbb{F}} Y\right)$ iff there are $x \in X$ and $y \in Y$ and there is $\mathcal{B} \subseteq \mathbb{F}$ such that $x \simeq_{\mathcal{B}} y$. Finally, define an elementary set (class) as

$$
x / \simeq_{\mathcal{B}}=\left\{x^{\prime} \in X \mid x^{\prime} \simeq_{\mathcal{B}} x\right\}
$$

and define a partition of $O$ (quotient set) as

$$
O / \simeq_{\mathcal{B}}=\left\{x_{/ \simeq_{\mathcal{B}}} \mid x \in O\right\} .
$$

A nearness measure (NM) useful in determining the degree of resemblance between two images is given in (22). Let the sets $X$ and $Y$ be weakly near each other in $\langle O, \mathbb{F}\rangle$, i.e., there exists $\mathcal{B} \subseteq \mathbb{F}$ such that $x \simeq_{\mathcal{B}} y$. Then, the degree of nearness between $X$ and $Y$ is measured using (22).

$$
N M_{\sim_{\mathcal{B}}}=\frac{\sum_{x / \simeq_{\mathcal{B}} \in X_{/ \sim_{\mathcal{B}}}} \sum_{y / \simeq_{\mathcal{B}} \in Y_{/ \sim_{\mathcal{B}}}} \eta\left(x_{/ \simeq_{\mathcal{B}}}, y_{/ \simeq_{\mathcal{B}}}\right)}{\max \left(\left|X_{/ \simeq_{\mathcal{B}}}\right|,\left|Y_{/ \simeq_{\mathcal{B}}}\right|\right)},
$$

where $\eta\left(x / \simeq_{\mathcal{B}}, y / \simeq_{\mathcal{B}}\right)$ in $(22)$ is defined as follows:

$$
\begin{aligned}
& \eta\left(x_{/ \sim_{\mathcal{B}}}, y / \sim_{\mathcal{B}}\right)= \\
& \begin{cases}\min \left(\left|x / \sim_{\mathcal{B}}\right|,\left|y / \sim_{\mathcal{B}}\right|\right) & , \text { if } \phi_{i}(x)=\phi_{i}(y) \forall \phi_{i} \in \mathcal{B}, \\
0 & , \text { otherwise. }\end{cases}
\end{aligned}
$$

In other words, the nearness of two sets can be measured by the cardinality of their elementary (equivalence) classes. Sets that are similar with respect to the probe functions in $\mathcal{B}$ will have equivalence classes with similar numbers of objects producing a nearness degree close to or equal to 1 . By contrast, sets that are not similar will have equivalence classes that share little with each other and will produce a nearness degree close to or equal to 0 .

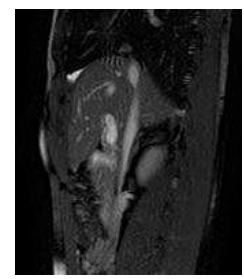

3.1: MRI image 1

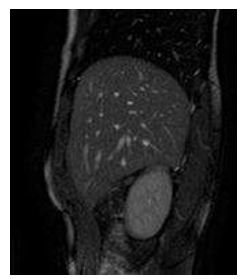

3.2: MRI image 2
Fig. 3. Sample MRI images of Respiratory Organ

The following simple example demonstrates these con-

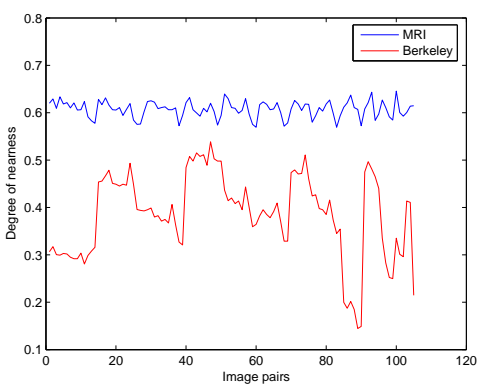

Fig. 4. Degree of nearness between image pairs.

cepts. Fifteen images from both the Berkeley Segmentation Dataset [78], and a 4DMRI dataset [79] were used to show that using this measure, similar images have a higher degree of nearness to each other than images which are not similar. In this example, the MRI images were used to represent images that are similar while the Berkeley Segmentation Dataset contains images which are of many different scenes and objects, and as such, do not have much relation to each other in terms of perceptual content of the images.

Formally, let $\mathbb{F}$ consist of a single probe function, namely the information content of the domain (input image). Further, let $X$ and $Y$ represent images that are partitioned into subimages, and let $O=X \cup Y$. Thus, each $o \in O$ is a perceptual object and in this example is given by a subimage of either image $X$ or $Y$. Thus, we have defined a perceptual information system $\langle O, \mathbb{F}\rangle$.

In this example, we are interested in the results of using Eq. 22 on images that are similar to each other versus images that are not. To this end, we performed two different experiments, one for the MRI dataset and one for the Berkeley dataset. For each experiment, we selected all unordered pairs of images and compared them using Eq. 22. This gave us $\left(\begin{array}{c}15 \\ 2\end{array}\right)=105$ comparisons. The results of this experiment are given in Fig. 4. As can be seen, this measure produces higher values for the MRI images which suggests that they are "nearer" each other than the Berkeley images. Also, there is less variability for the MRI values because the Berkeley images are quite different from each other. These results are promising and will lead to future work in object recognition using the near set approach.

\section{ViI. Promising Generalizations of Rough Sets in MEDICAL IMAGING}

In addition to near sets, this section briefly considers a number ofother generalizations of rough sets that have promise in medical image analysis. This section brief presents the basic approach in several of these generalizations, namely, tolerance spaces [80], [81], [82], [83], [84], [85], neighbourhood systems [86], [87], [88], and shadowed sets [89], [90], [91].

\section{A. Tolerance Spaces and Neighbourhood Systems}

The term tolerance space was coined by E.C. Zeeman in 1961 in modelling visual perception with tolerances [80]. 
Images are viewed as sets of fixed points. Let $O$ denote a set of perceptual objects (e.g., grey level images) and let $\simeq$ denote a relation that is reflexive (for all $x \in O, x \simeq x$ ) and symmetric (for all $x, y \in O, x \simeq y$ and $x \simeq y$ ) but transitivity of $\simeq$ is not required. Then $(O, \simeq)$ is a tolerance space. A tolerance is directly related to the exact idea of closeness or resemblance or being within tolerance in comparing objects. The basic idea is to find objects such as images that resemble each other with a tolerable level of error. The main idea underlying tolerance theory comes from Henri Poincaré [92]. Physical continuum (e.g., measurable magnitudes in the physical world of medical imaging) are contrasted with the mathematical continuum (real numbers) where almost solutions are common and a given equation have no exact solutions. An almost solution of an equation (or a system of equations) is an object which, when substituted into the equation, transforms it into a numerical 'almost identity', i.e., a relation between numbers which is true only approximately (within a prescribed tolerance) [81]. Equality in the physical world is meaningless, since it can never be verified either in practice or in theory. Hence, the basic idea in a tolerance view of medical imaging, for example, is to replace the indiscerniblity relation in rough sets with a tolerance relation.

For example, tolerance near sets were introduce in [84] and elaborated in [85], [83]. Briefly, here is the basic approach.

Definition 4: Tolerance Relation Let $\langle O, \mathbb{F}\rangle$ be a perceptual system and let $\in \in \mathbb{R}$ (set of all real numbers). For every $\mathcal{B} \subseteq \mathbb{F}$ the tolerance relation $\cong_{\mathcal{B}}$ is defined as follows:

$$
\cong_{\mathcal{B}, \epsilon}=\{(x, y) \in O \times O:\|\phi(x)-\phi(y)\| \leq \epsilon\} .
$$

If $\mathcal{B}=\{\phi\}$ for some $\phi \in \mathbb{F}$, instead of $\cong_{\{\phi\}}$ we write $\cong_{\phi}$. Further, for notational convince, we will write $\cong_{\mathcal{B}}$ instead of $\cong_{\mathcal{B}, \epsilon}$ with the understanding that $\epsilon$ is inherent to the definition of the tolerance relation.

As in the case with the indiscernibility relation, a tolerance class can be defined as

$$
x / \cong_{\mathcal{B}}=\left\{y \in X \mid y \cong_{\mathcal{B}} x\right\} .
$$

Notice that Defn. 4 does not uniquely partition $O$ (i.e. an object can belong to more than one class). For this reason, (23) is called a tolerance class instead of an elementary set. In addition, each pair of objects $x, y$ in a tolerance class $x / \cong_{\mathcal{B}}$ must satisfy the condition $\|\phi(x)-\phi(y)\| \leq \epsilon$. Next, a quotient set for a given a tolerance relation is the set of all tolerance classes and is defined as

$$
O / \cong_{\mathcal{B}}=\left\{x_{/ \cong_{\mathcal{B}}} \mid x \in O\right\} .
$$

As was the case with the equivalence relation, tolerance classes reveal relationships in perceptual systems leading to the definition of a tolerance nearness relation.

Definition 5: Weak Tolerance Nearness Relation [84] Let $\langle O, \mathbb{F}\rangle$ be a perceptual system and let $X, Y \subseteq O, \epsilon \in$ $\mathbb{R}$. The set $X$ is perceptually near to the set $Y$ within the perceptual system $\langle O, \mathbb{F}\rangle\left(X \unrhd_{\mathbb{F}} Y\right)$ iff there exists $x \in X$,

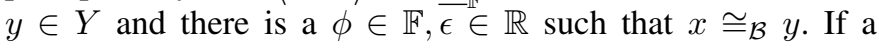
perceptual system is understood, then we say shortly that a set $X$ is perceptually near to a set $Y$ in a weak tolerance sense of nearness.

A tolerance nearness measure (tNM) under a tolerance relation is given as

$$
t N M_{\cong_{\mathcal{B}}}=\sum_{x / \simeq_{\mathcal{B}} \in X / \simeq_{\mathcal{B}}} \sum_{y / \simeq_{\mathcal{B}} \in Y_{/ \simeq_{\mathcal{B}}}} \frac{\xi\left(x / \simeq_{\mathcal{B}}, y / \cong_{\mathcal{B}}\right)}{\max \left(\left|x / \cong_{\mathcal{B}}\right|,\left|y / \sim_{\mathcal{B}}\right|\right)},
$$

where

$$
\begin{aligned}
\xi\left(x / \cong_{\mathcal{B}}, y / \cong_{\mathcal{B}}\right)= & \begin{cases}\min \left(\left|x / \cong_{\mathcal{B}}\right|,\left|y / \cong_{\mathcal{B}}\right|\right) & , \text { if }\|\phi(x)-\phi(y)\| \leq \epsilon, \\
0 & , \text { otherwise }\end{cases}
\end{aligned}
$$

Notice the subtle difference between the two nearness measures, namely, NM in (22) and tNM in (24). Since objects can belong to more than one tolerance class, the denominator of Eq. 24 has moved inside the summation. Similarly, Eq.'s $22 \& 24$ are equivalent when $\epsilon=0$.

The neighbourhood system (NS) paradigm has been widely used in image analysis [93], [94], [95], [96]. Neighbourhood systems were introduced by Sierpenski and Krieger during the mid-1950s [86], adopted by T.Y. Lin during the late 1980s for describing relationships between objects in database systems [87] and considered in the context of rough sets [97], [88]. Associated with a neighbourhood system (NS) is a set of cliques. A clique is either a single site or subset of sites such than any two sites are neighbours of each other [98]. Cliques are uniquely determined by the particular NS chosen. For an element $x$ in a finite universe $U$, one associates a neighbourhood $B(x) \subseteq U$. A $N S(x)$ is a nonempty family of neighbourhoods of $x$ [88].

\section{B. Shadowed Sets}

Shadowed sets (ShS) were introduced in 1998 by Witold Pedrycz as a means of simplifying processes carried out with fuzzy sets and in establishing a bridge between rough sets and fuzzy sets [89] and in image processing [90], [91]. A shadowed set is viewed as an approximation of a fuzzy set [89], [99]. A shadowed set is a localization of membership values by forming "shadows" and using only 0-1 degrees of membership. Let $A \subseteq X$ (i.e., A is a subset of the universe of discourse $X$ ) and let $\mu_{A}: X \rightarrow[0,1]$ (i.e., $\mu_{A}$ is a membership function associated with the fuzzy set $A$. In Fig. 5, the membership values belonging to $(\alpha, 1-\alpha)$ are those values characterized by great uncertainty or lack of knowledge and they are considered the "shadow" of the induced shadowed set. In general, a shadowed set on $X$ is any mapping $s: X \rightarrow\{0,1,(0,1)\}$. For the sample shadowed set in Fig. 5, let $\alpha \in(0,0.5)$ be a fixed value, then the $\alpha$-shadowed set of $\mu_{A}$ (denoted by $s_{\alpha}\left(\mu_{A}\right)$ is defined to be a shadow of $A$ [100], where

$$
s_{\alpha}\left(\mu_{A}\right)(x)=\left\{\begin{array}{l}
0, \text { if } \mu_{A}(x) \leq \alpha \\
1, \text { if } \mu_{A}(x) \geq 1-\alpha, \\
0.5, \text { otherwise }
\end{array}\right.
$$

\section{Choosing a Technology for Medical Image Analysis}

Rough sets are ideally suited for feature-based image segmentations,image clustering and approximations of medical 


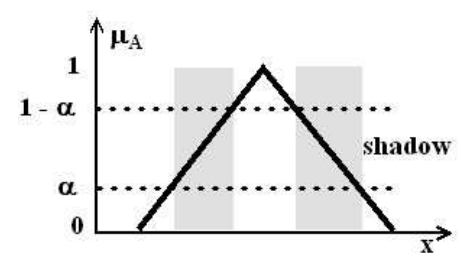

Fig. 5. Sample shadowed set [89]

images. Pal's rough image entropy model is very useful in classifying images relative to the information content of either image regions or entire images and in extracting objects from greyscale images. Notice that the focus in the rough set approach to medical imaging is on approximation methods applied to single images or in grouping parts of an image in terms of equivalence classes. In applications where there is a need to determine the degree of resemblance (nearness) between medical images and to find clusters of medical images that resemble each other, then tolerance spaces in general and tolerance near sets, in particular, are useful.

The conjecture here is that neighbourhood systems will be useful in analyzing image sequences found in video microscopy, X-ray cinematrography, 3D laser-scanning cofocal microscopy (LSCM) and magnetic resonance imaging (MRI), where there is an interest in observing shape-change and extracting meaningful information from image sequences. Rather than global information (how a specimen has translated, rotated or changed as a whole, to what extent pairs of images resemble each other) is easily detected using tolerance near sets, whereas neighbourhood systems are more suited for extracting local information about shape-change of individual regions within a specimen.

An obvious advantage to shadowed sets is a simplified view of fuzzy sets in medical image analysis. The side-effect of introducing a shadowed set is shifting attention to an $\alpha$-region of a fuzzy set considered important for a particular application such as medical imaging.

\section{Challenges and Future DiRECTIONS}

Rough set theory encompasses an extensive set of $C I$ based methods that have been applied in the medical domain and that are used for the discovery of data dependencies, importance of features, patterns in sample data, and feature space dimensionality reduction. Most of the current literature on rough set-based methods for medical imaging focuses on classification and dimensionality reduction issues. A number of papers also deal with medical imaging problems such as image segmentation, image filtering, and voxel representation. From what has been presented in the literature, it is obvious that the rough set approach provides a promising means of solving a number of medical imaging problems. It should be observed that rough set or near set by themselves or in combination with other $C I$ technologies work remarkably well in partitioning medical images into approximation regions that facilitate automated image segments and object recognition. The challenge now is to develop near sets-based methods that offer an approach to classifying perceptual objects by means of features. It is fairly apparent that near set methods can be useful in object recognition, especially in solving medical imaging problems. The near set approach to object description, feature selection, and automatic image segmentation based on the partition of an image into equivalence classes offer a practical as well as straightforward approach to classifying images. It is in the domain of medical image segmentation that the near set approach holds the greatest promise for medical imaging.

A combination of various computational intelligence technologies in pattern recognition and, in particular, medical imaging problems has become one of the most promising avenues in image processing research. From the perspective of rough sets, further explorations into possible hybridization of rough sets with other $C I$ technologies are necessary to build a more complete picture of rough or near set-based applications in medical imaging. What can be said at this point is that the rough set and near set approaches pave the way for new and interesting avenues of research in medical imaging and represents an important challenge for $C I$ researchers.

\section{REFERENCES}

[1] J. Bezdek, "What is computational intelligence?" in Computational Intelligence: Imitating Life, J. Zurada, R. Marks, and C. Robinson, Eds. Piscataway, NJ: IEEE Press, 1994, pp. 1-12.

[2] W. Pedrycz, Computational Intelligence: An Introduction. Boca Raton, FL: CRC Press, 1998.

[3] J. Peters and W. Pedrycz, "Computational intelligence," in Electrical \& Electronics Engineering Encyclopedia. NY: John Wiley \& Sons, Ltd, 2008.

[4] A. Hassanien, "Classification and feature selection of breast cancer data based on decision tree algorithm," Int. J. of Studies in Informatics and Control, vol. 12, no. 1, pp. 33-39, 2003.

[5] A. Hassanien and J. Ali, "Image classification and retrieval algorithm based on rough set theory," South African Computer Journal, vol. 30, pp. 9-16, 2003.

[6] N. Karssemeijer, M. Thijssen, J.Hendriks, and E. L. Van Erning, Digital Mammography: Computational Imaging and Vision. The Netherlands: Kluwer, 1998.

[7] R. Setiono, "Generating concise and accurate classification rules for breast cancer diagnosis," Artificial Intelligence in Medicine, vol. 18, no. 3, pp. 205-219, 2000.

[8] S. Das, A. Abraham, and S. Sarkar, "A hybrid rough set-particle swarm algorithm for image pixel classification," in Proc. of the Sixth Int. Conf. on Hybrid Intelligent Systems, 2006, pp. 26-32.

[9] Y. Yao, "A comparative study of fuzzy sets and rough sets," Journal of Information Sciences, vol. 109, pp. 227-242, 1998.

[10] S. Tsumoto, "Mining diagnostic rules from clinical databases using rough sets and medical diagnostic model," Information Sciences: An International Journal, vol. 162, no. 2, pp. 65-80, 2004.

[11] S. Widz, D. Ślęzak, and K. Revett, "Application of rough set based dynamic parameter optimization to mri segmentation," in IEEE Annual Meeting of the Information Processing NAFIPS, vol. 1, 2004, pp. 440445.

[12] R. Swiniarski, "Rough sets and bayesian methods applied to cancer detection," in First Int. Conf. on Rough Sets and Current Trends in Computing, 1998, pp. 609-616.

[13] Z. Pawlak, "Classification of objects by means of attributes," Polish Academy of Sciences, vol. 429, 1981.

[14] Z. Pawlak and A. Skowron, "Rudiments of rough sets," Information Sciences, vol. 177, pp. 3-27, 2007.

[15] — "Rough sets: Some extensions," Information Sciences, vol. 177, pp. 28-40, 2007.

[16] - "Rough sets and boolean reasoning," Information Sciences, vol. 177, pp. 41-73, 2007.

[17] J. Peters and A. Skowron, Transactions on Rough Sets, vol. I-IX, 20042008.

[18] J. Peters, "Near sets. special theory about nearness of objects," Fundamenta Informaticae, vol. 75, no. 1-4, pp. 407-433, 2007. 
[19] — - "Near sets. toward approximation space-based object recognition," Lecture Notes in Artificial Intelligence, vol. 4481, pp. 22-33, 2007

[20] — - "Near sets. general theory about nearness of objects," Applied Mathematical Sciences, vol. 1, no. 53, pp. 2609-2029, 2007.

[21] J. Peters, A. Skowron, and J. Stepaniuk, "Nearness of objects: Extension of approximation space model," Fundamenta Informaticae, vol. 79, no. 3-4, pp. 497-512, 2007.

[22] J. Peters and S. Ramanna, "Feature selection: Near set approach," in Int. Workshop on Mining Complex Data, ECML/PKDD, LNAI 4944, Warsaw, Poland, 2007, pp. 57-72.

[23] J. Peters, "Classification of perceptual objects by means of features," Int. J. of Info. Technology \& Intelligent Computing, vol. 3, no. 2, pp. $1-35,2008$.

[24] — "Classification of objects by means of features," in Proc. IEEE Symposium Series on Foundations of Computational Intelligence (IEEE SCCI 2007), Honolulu, Hawaii, 2007, pp. 1-8.

[25] C. Henry and J. Peters, "Near set image segmentation quality index," in GEOBIA 2008 Pixels, Objects, Intelligence. GEOgraphic Object Based Image Analysis for the 21st Century, University of Calgary, Alberta, 2008, pp. 1-6.

[26] — " "Image pattern recognition using approximation spaces and near sets," in Proc. 11th Int. Conf. on Rough Sets, Fuzzy Sets, Data Mining and Granular Computing (RSFDGrC 2007), Joint Rough Set Symposium (JRS 2007), Lecture Notes in Artificial Intelligence 4482, Heidelberg, Germany, 2007, pp. 475-482.

[27] J. Peters, "Perceptual granulation in ethology-based reinforcement learning," in Handbook on Granular Computing, W. Pedrycz, A. Skowron, and V. Krinovich, Eds. West Sussex, England: John Wiley \& Sons, Ltd, 2008, pp. 671-689.

[28] J. Peters, S. Shahfar, S. Ramanna, and T. Szturm, "Biologically-inspired adaptive learning: A near set approach," in Frontiers in the Convergence of Bioscience and Information Technologies, Jeju City, Korea, 2007, pp. 403-408

[29] S. Hirano and S. Tsumoto, "Segmentation of medical images based on approximation in rough set theory," in Proc. 11th Int. Conf. on Rough Sets and Current Trends in Computing, Lecture Notes in Artificial Intelligence 2475, Heidelberg, Germany, 2002, pp. 554-563.

[30] Z. Wojcik, "Rough approximation of shapes in pattern recognition," Comput. Vision Graphics Image Process, vol. 40, no. 2, pp. 228-249, 1987.

[31] Z. Yuan, Y. Wu, G. Wang, and J. Li, "Motion-information-based video retrieval system using rough pre-classification," Transactions on Rough Sets, vol. V, pp. 306-333, 2006.

[32] J. Jelonek, K. Krawiec, R. Slowinski, and J. Stefanowski, "Rough set reduction of features for picture-based reasoning," in Advances in Fuzzy Systems, T. Lin, Ed., 1995, pp. 89-92.

[33] A. Mohabey and A. Ray, "Rough set theory based segmentation of color images," in Proc. of NAFIPS 19th Int. Conf., 2000, pp. 338-342.

[34] D. Sinha and P. Laplante, "A rough set-based approach to handling spatial uncertainty in binary images," Eng. Appl. Artif. Intell., vol. 17, pp. 97-110, 2004.

[35] S. Pal, "Fuzzy image processing and recognition: Uncertainties handling and applications," Int. J. Image Graphics, vol. 1, no. 2, pp. 169195, 2001.

[36] S. Pal, B. U. Shankar, and P. Mitra, "Granular computing, rough entropy and object extraction," Pattern Recognition Letters, vol. 26, no. 16 , pp. 2509-2517, 2005.

[37] S. Hirano and S. Tsumoto, "Rough representation of a region of interest in medical images," Int. J. of Approximate Reasoning, vol. 40, pp. 23 34, 2005.

[38] J.Liang and Z. Shi, "The information entropy, rough entropy and knowledge granulation in rough set theory," Int. J. of Uncertainty, Fuzziness and Knowledge-Based Systems, vol. 12, no. 1, pp. 37-46, 2004.

[39] P. Lingras, M. Hogo, and M. Snorek, "Interval set clustering of web users using modified kohonen self-organizing maps based on the properties of rough sets," Web Intelligence and Agent Systems: An International Journal, vol. 2, no. 3, pp. 217-225, 2004.

[40] C.-B. Chena and L.-Y. Wang, "Rough set-based clustering with refinement using shannon's entropy theory," Computers and Mathematics with Applications, vol. 52, no. 10-11, pp. 1563-1576, 2006.

[41] M. Ahmed, S. Yamany, M. Nevin, and A. Farag, "A modified fuzzy c-means algorithm for bias field estimation and segmentation of mri data," IEEE Transactions on Medical Imaging, vol. 21, no. 3, pp. 193199,2003
[42] P. Lingras, "Applications of rough set based k-means, kohonen, ga clustering," Transactions on Rough Sets, vol. VII, pp. 217-225, 2007.

[43] M. Yan and J. Karp, "Segmentation of 3d brain mr using an adaptive k-means clustering algorithm," in IEEE Conf. on Nuclear Science Symposium and Medical Imaging, vol. 4, 1994, pp. 1529 - 1533.

[44] H. Ng, S. Ong, K. Foong, P. Goh, and W. Nowinski, "Image segmentation using k-means clustering and improved watershed algorithm," in IEEE Southwest Symposium on Image Analysis and Interpretation, 2006, pp. 61-65.

[45] C. Chen, J. Luo, and K. Parker, "Image segmentation via adaptive kmeans clustering and knowledge-based morphological operations with biomedical applications," IEEE Trans. on Image Processing, vol. 7, no. 12, pp. 1673-1683, 1998.

[46] C. Shang and Q. Shen, "Rough feature selection for neural network based image classification," Int. J. of Image and Graphics, vol. 2, no. 4 , pp. 541-555, 2002.

[47] N. Zhang, N. Tomuro, D. Raicu, and J. Furst, "Investigation on feature selection to improve classification of abdominal organs in ct images,' in CTI Research Symposium, 2006, pp. 1287-1296.

[48] R. Swiniarski and L. Hargis, "Rough sets as a front end of neuralnetworks texture classifiers," Neurocomputing, vol. 36, no. 1-4, pp. 85-102, 2001.

[49] Q. Hu, D. Yu, and Z. Xie, "Information-preserving hybrid data reduction based on fuzzy-rough techniques," Pattern Recognition Letters, vol. 27, pp. 414-423, 2006.

[50] M. Zorman, P. Kokol, M. Lenic, L. de la R.J. Francisco, and S. Alayon, "Symbol-based machine learning approach for supervised segmentation of follicular lymphoma images," in Proc. of the Twentieth IEEE Int. Symp. on Computer-Based Medical Systems, 2007, pp. 115-120.

[51] K. Thangave, M. Karnan, and A. Pethalakshmi, "Performance analysis of rough reduct algorithms in image mammogram," ICGST International Journal on Graphics, Vision and Image Processing, vol. 8, pp. 13-21, 2005.

[52] K. Cyran and A. Mrozek, "Rough sets in hybrid methods for pattern recognition," Int. J. of Intelligent Systems, vol. 16, no. 2, pp. 149-168, 2001.

[53] J. Yun, L. Zhanhuai, W. Yong, and Z. Longbo, "Joining associative classifier for medical images," in Fifth Int. Conf. on Hybrid Intelligent Systems, 2005, pp. 367-372.

[54] A. Hassanien and D. Selzak, "Rough neural intelligent approach for image classification: A case of patients with suspected breast cancer," International Journal of Hybrid Intelligent Systems, vol. 3, no. 4, pp. 205-218, 2006.

[55] X. Wanga, J. Yanga, R. Jensen, and X. Liua, "Rough set feature selection and rule induction for prediction of malignancy degree in brain glioma," Information Processing \& Management, vol. 8, no. 3, pp. 147-156, 2006.

[56] A. Leticia, B. Rafael, and M. Garcia, "Image pattern recognition using approximation spaces and near sets," in Proc. of the Fifth Mexican Int. Conf. on Artificial Intelligence, 2006, pp. 168-177.

[57] S. Pal and R. King, "Histogram equalisation with $s$ and ii functions in detecting x-ray edges," IEE Electronics Letters, vol. 17, no. 8, pp. 302-304, 1981.

[58] _ , "Image enhancement using smoothing with fuzzy sets," IEEE Trans. Systems, Man, and Cybernetics, vol. SMC-11, no. 7, pp. 494501, 1981.

[59] S. Pal, "A note on the quantitative measure of image enhancement through fuzziness," IEEE Trans. Pat. Anal. \& Mach. Intell., vol. PAMI4, no. 2, pp. 204-208, 1982.

[60] A. Hassanien and J. Ali, "Rough set approach for classification of breast cancer mammogram images," Lecture Notes in Computer Science, vol. 2955, pp. 224-231, 2006.

[61] Q. Shen and A. Chouchoulas, "A rough-fuzzy approach for generating classification rules," Pattern Recog., vol. 35, no. 11, pp. 2425-2438, 2002.

[62] P. Lingras, "Rough neural networks," in Proc. of the 6th Int. Conf. on Information Processing and Management of Uncertainty in Knowledgebased Systems, Granada, Spain, 1996, pp. 1445-1450.

[63] J. Peters, L. Han, and S. Ramanna, "Rough neural computing in signal analysis," Computational Intelligence, vol. 17, no. 3, pp. 493-513, 2001.

[64] J. Peters, A. Skowron, L. Han, and S. Ramanna, "Towards rough neural computing based on rough membership functions: Theory and application," in Proc. of Rough Sets and Current Trends in Computing, LNAI 2005, 2000, pp. 611-618 
[65] F. Chiang and R. Braun, Intelligent Failure Domain Prediction in Complex Telecommunication Networks with Hybrid Rough Sets and Adaptive Neural Nets, 2004.

[66] J. Yun, L. Zhanhuai, W. Yong, and Z. Longbo, "A better classifier based on rough set and neural network for medical images," in Proc. of the Sixth IEEE Int. Conf. on Data Mining, 2006, pp. 853-857.

[67] P. Srinivasan, M. Ruiz, D. Kraft, and J. Chen, "Vocabulary mining for information retrieval: rough sets and fuzzy sets," Information Processing \& Management, vol. 37, no. 1, pp. 15-38, 1998.

[68] C.-W. Mao, S.-H. Liu, and J.-S. Lin, "Classification of multispectral images through a rough-fuzzy neural network," Optical Engineering, vol. 43, no. 1, pp. 103-112, 2004.

[69] X. Wang, J. Yang, X. Teng, and N. Peng, "Fuzzy classifiers fuzzy rough set based nearest neighbor clustering classification algorithm," Lecture Notes in Computer Science, vol. 3613, pp. 370-373, 2005.

[70] A. Hassanien, "Fuzzy-rough hybrid scheme for breast cancer detection," Image and Computer Vision Journal, vol. 25, no. 2, pp. 172-183, 2006.

[71] S. Mitra, H. Banka, and W. Pedrycz, "Rough-fuzzy collaborative clustering systems," IEEE Transactions on Man and Cybernetics, Part B: Cybernetics, vol. 36, no. 4, pp. 795-805, 2006.

[72] A. Petrosino and G. Salvi, "Rough fuzzy set based scale space transforms and their use in image analysis," Int. J. of Approximate Reasoning, vol. 41, no. 2, pp. 212-228, 2006.

[73] M. Sarkar, "Rough-fuzzy functions in classification," Fuzzy Sets and Systems, vol. 132, no. 3, pp. 353-369, 2002.

[74] G. Zhang, Z. Cao, and Y. Gu, "A hybrid classifier based on rough set theory and support vector machines," Lecture Notes in Computer Science, vol. 3613, pp. 306-333, 2005.

[75] P. Lingras and C. Butz, "Rough set based 1-v-1 and 1-v-r approaches to support vector machine multi-classification," Information Sciences: an International Journal, vol. 177, no. 18, pp. 3782-3798, 2007.

[76] B. Qiu, C. Xu, and Q. Tian, "An automatic classification system applied in medical images," in IEEE Int. Conf. on Multimedia and Expo, 2006, pp. $1045-1048$.

[77] Y. Jiang, Z. Li, L. Zhang, and P. Sun, "An improved svm classifier for medical image classification," in Int. Conf. on Rough Sets and Emerging Intelligent Systems Paradigms, LNAI, vol. 4585, 2007, pp. 764-773.

[78] D. Martin, C. Fowlkes, D. Tal, and J. Malik, "A database of human segmented natural images and its applications to evaluating segmentation algorithms and measuring ecological statistics," in Proc. of the 8th International Conference on Computer Vision, vol. 2, Vancouver, B.C., 2001, pp. 416-423.

[79] M. von Siebenthal. (2005) Respiratory organ motion from 4dmri. [Online]. Available: http://www.vision.ee.ethz.ch/4dmri/

[80] E. Zeeman, "The topology of the brain and visual perception," University of Georgia Institute, 1961, published in M.K. Fort, Jr. (Ed.), Topology of 3-Manifolds and Related Topics, Prentice-Hall, Inc., 1961, 240-256.

[81] A. B. Sossinsky, "Tolerance space theory and some applications," Acta Applicandae Mathematicae: An International Survey Journal on Applying Mathematics and Mathematical Applications, vol. 5, no. 2, pp. 137-167, 1986.

[82] A. Skowron and J. Stepaniuk, "Tolerance approximation spaces," Fundamenta Informaticae, vol. 27, pp. 245-253, 1996

[83] C. Henry and J. Peters, "Perception based image classification," IEEE Trans. Systems, Man and Cybernetics, Part B: Cybernetics, 2008, submitted, revised, resubmitted.

[84] J. Peters, "Discovery of perceptually near information granules," in Novel Developments in Granular Computing: Applications for Advanced Human Reasoning and Soft Computation, J. Yao, Ed. Hershey, NY: Information Science Reference, 2008, to appear.

[85] J. Peters and S. Ramanna, "Affinities between perceptual granules: Foundations and perspectives," in Human-Centric Information Processing Through Granular Modelling, A. Bargiela and W. Pedrycz, Eds. Berlin: Springer-Verlag, 2008, to appear.

[86] W. Sierpenski and C. Krieger, General Topology. Toronto, ON: University of Toronto, 1956.

[87] T. Lin, "Neighborhood systems and approximation in relational databases and knowledge bases," in Proc. 4th International Symposium on Methodologies of Intelligent Systems, 1988, pp. 75-86.

[88] Y. Yao, "Rough sets, neighborhood systems, and granular computing," in Proc. IEEE Canadian Conf. on Electrical and Computer Engineering, 1999, pp. 1553-1558.

[89] W. Pedrycz, "Shadowed sets: representing and processing fuzzy sets," IEEE Trans. on Systems, Man, and Cybernetics, Part B: Cybernetics, vol. 28, no. 1, pp. 103-109, 1998.
[90] — - "Shadowed sets: sets: bridging fuzzy and rough sets," in RoughFuzzy Hybridization: A New Trend in Decision Making, 1999, pp. 179199.

[91] B. Barman, S. Mitra, and W. Pedrycz, "Shadowed clustering for speech data and medical image segmentation," in Proc. of the 6th Int. Conf. on Rough Sets \& Current Trends in Computing, 2008, pp. 475-484.

[92] H. Poincaréa, Mathematics and Science: Last Essays, 1913, trans. J.W. Bolduc. Kessinger Pub.: NY, 2007.

[93] H. Choi and C. Chung, "An image model for quantitative image analysis," in Proc. Int. Conf. on Image Processing, vol. 3, 1996, pp. $16-19$.

[94] J. S. Suri, D. L. Wilson, and S. Laxminarayan, Handbook of Biomedical Image Analysis. Berlin: Springer, 2005.

[95] J. Pastore, A. Bouchet, E. Moler, and V. Ballarin, "Topological concepts applied to digital image processing," JCS \& T, vol. 6, no. 2, pp. 80-84, 2007.

[96] H. Ning, Z. Minhui, and Z. Shourong, "Considering neighborhood information in image fuzzy clustering," Journal of Electronics, vol. 19, no. 3, pp. 307-310, 2006.

[97] T. Lin, "Granular computing on binary relations i: data mining and neighborhood systems, ii: rough set representations and belief functions," in Rough Sets in Knowledge Discovery 1, L. Polkowski and A. Skowron, Eds. Heidelberg: Physica-Verlag, 1998, pp. 107-140.

[98] E. D.-P. Häder, Image Analysis. CRC Press: Berlin, 1992.

[99] R. Jensen, "Combining rough and fuzzy sets for feature selection," Ph.D. dissertation, School of Informatics, 2005. [Online]. Available: http://users.aber.ac.uk/rkj/PhDthesis.pdf

[100] G. Cattaneo and D. Ciucci. (2008, nov) Shadowed sets and related algebraic structures. [Online]. Available: http: www.fislab.disco.unimib.it/media/people/fi55.pdf 